\title{
Building Unstructured Crime Data Prediction Model (Practical Approach)
}

\author{
Mona Mowafy ${ }^{1}$, A. Rezk ${ }^{2}$, H. M. El-bakry ${ }^{3}$
}

\#1 Faculty of Computers and Information, Mansoura University, Kafr EL Sheikh, 33512, Egypt,mm_is_85@yahoo.com

\#2 Faculty of Computers and Information, Mansoura University, Mansoure, 35516, Egypt, amira_rezk@mans.edu.eg

\#3 Faculty of Computers and Information, Mansoura University, Mansoura, 35516, Egypt, helbakry5@yahoo.com

\section{ABSTRACT}

In the most of police stations, it's essential to classify the crimes, according to their types before applying the methods of pattern identification on the crime data. Also the legal organizations aim to divide the crimes into categories for various purposes related to the courts' procedure, such as assigning different types of court to different type of crimes. As a result, the crime type prediction is a decisive part of a crime analysis. There is a little research, in methods and techniques that can predict the crime type from unstructured text. This paper, focuses on how practically building an unstructured crime data prediction model using the scikit-learn Python Toolkit. The main goal of the constructed model is predicting the type of the crime, according to the unstructured data of the crime incident reports for improving the policeman decisions and reduce their investigation efforts..

Key words: Crime data prediction, Unstructured data prediction, practical crime prediction model.

Corresponding Author: Mona Mowafy

\section{1- INTRODUCTION}

Criminology is the scientific study of the non-legal portions of crimes, including its characteristics, causes, correction, prevention, the behavior of the criminal involved in the crime, and the social impact of crime [1]. The process of crime analysis is considered an important part of the criminology. In this process all the police report as reviewed every day with the goal of identifying patterns and hotspots that provide the law enforcement and intelligence-gathering organizations with important information such as increasing or decreasing of a particular type of crime, the percentage rate of a particular crime type, the area in which minimum or maximum crime happends, and the time when crime less of mostly happens etc. This information can be used to solve the complicated crimes, develop effective strategies to prevent or at least reduce the future crimes, find and catch offenders.

Crime type prediction is a critical part of a criminal analysis. The successful prediction assists the police in tracing the source of crimes, which can facilitate the inferring of the crime trends from various criminal activities and fight against criminal activities on time. Usually, 
DOI: https://dx.doi.org/10.26808/rs.ca.i8v4.01 International Journal of Computer Application (2250-1797)

Issue 8 Volume 4, July-August 2018

the reports about crimes are done manually and is in unstructured form. Analyzing the crimes manually from this unstructured data is time consuming consuming due to the complexity of issues. The investigators can take months to solve a crime puzzle.

Mose of publications and research projects in this area, include applying the tools and software on structured data. This paper aims to construct unstructured crime data prediction model to predict the type of the crime using Scikit-Learn.

The organization of this paper is as follows: section 2 covers the related work of using the text mining technique in the crime analysis, section 3 describes the selected text classification model, section 4 illustrates the methodology and results of building the model, and finally section 5 presents the conclusions.

\section{2- RELATED WORK}

There are a lot of tools and softwares which the researchers used in their studies of crime mining applications. Waseem Ahmed, and et al. [2] have used a Rapid Miner as a data mining tool and Naive Bayes as a classifier algorithm to classify the different types of crime. The authors have demonstrated the efficiency of Naive Bayes for managing this data with a good result by applying the classification algorithm on the crime data using the Rapid Miner. Rasoul Kiani, and et al. [3] have used a Rapid Miner tool for applying a theoretical model based on classifying the clustered crimes of real. They have used the Genetic algorithm (GA) for optimizing of Outlier Detection operator parameters.

Zakaria Zubi, and et al. [4] have presented a proposed model using the Weka tool for analyzing crime and criminal data based on clustering technique using k-means algorithm and classifying technique using association rules algorithm. Obuandike Georgina N., and et al [5] have used Weka as a machine learning tool to measure the performance of some of classification techniques such as J48 as a type of decision tree classifier, Naive Bayes classifier that applies probability functions, and ZeroR which is a rule induction classifier.

Tahani Almanie, and et al. [6] have applied a priori algorithm to find frequent crime patterns. They also have used Scikit-Learn for applying the decision tree and Naive Bayes in order to predict the potential crime types.

The crime prediction is considered one of the main tasks of the crime mining process. Most of publications and research studies in this field focuses on the structured data of the police departments. Somayeh, Aida, and et al. [7] aimed to classify experimental structured dataset into two categories which are critical and non - critical. They have used five classification algorithms using two ways of feature selection techniques, manually and chi square, to determine more accurate classifiers. The experiment results have shown that k-nearest neighbor algorithm using chi square achieved the best accuracy.

Noora Abdulrahman and Wala Abedalkhader [8] have applied two of data mining techniques - K-nearest neighbor and Naive Bayes to identify and then predict the classification of a crime based on the time and location attributes of the structured dataset of San Francisco. The experimental results have shown that a higher classification accuracy can be obtained by using the Multinomial Naive Bayes.

Cuicui, Chunlong, and et al. [9] have proposed a new data filling algorithm, which called GMKNN to improve the classification accuracy for predicting crime types. The proposed algorithm based on combing Mutual K-nearest neighbor algorithm with Grey system theory. The proposed GMKNN algorithm increases the classification accuracy to 77,837\%. Cuicui, Chunlong, and et al [10] have used three filling algorithms - Maximum class filling algorithm, Roulette algorithm and GBWKNN algorithm to fill the real crime dataset to obtain the complete dataset. Finally the classification accuracy of three algorithms, including C4.5 algorithm, Naive Bayes algorithm, and K-nearest neighbor algorithm, are compared based 
the completed dataset. The highest accuracy is $66.6939 \%$, which is achieved by combining GBWKNN filling algorithm and K-nearest neighbor classification algorithm.

\section{3- THE UNSTRUCTURED CRIME DATA PREDICTION}

Crime type prediction is effectively achieved using the classification techniques. Our Model of crime prediction applies the unstructured text classification model that has been proposed in [11]. This classification model is basically depends on combining the Multinomial Naive Bayes as a classification technique, and TF-IDF as a vector space model for text extraction for more accurate text classification results. The selected model as in figure 2 is responsible for classifying the unstructured data.

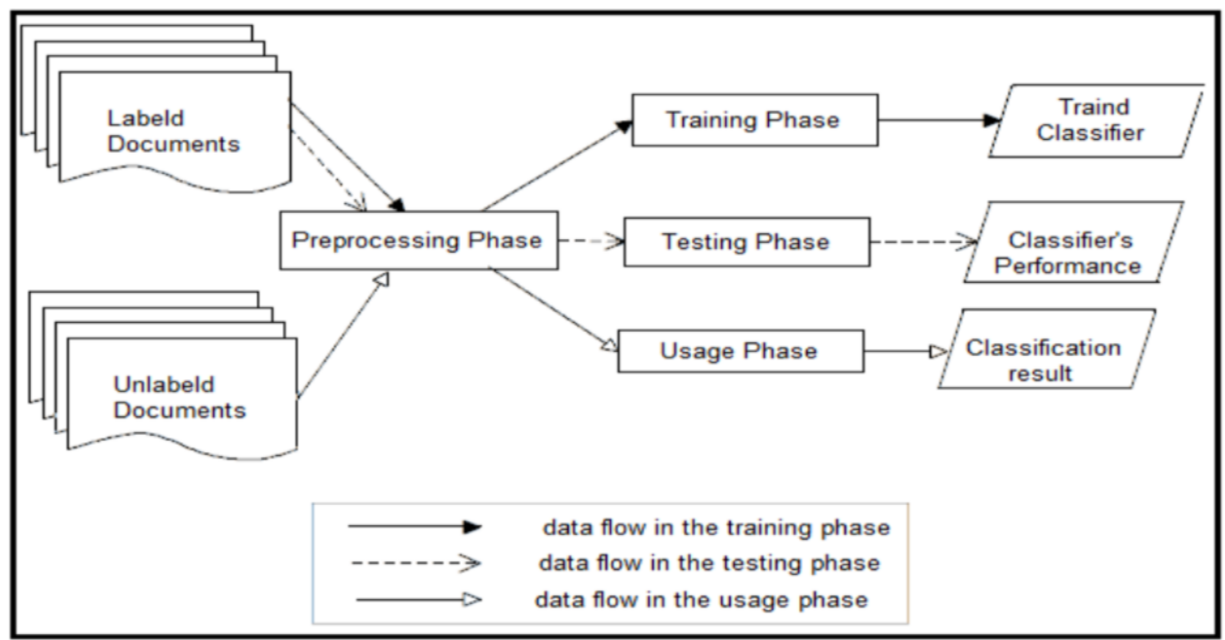

Figure 1: The architecture of the classification model of the unstructured text documents [11]

\section{4- PRACTICAL STEPS OF UILDING THE MODEL OF CRIME PREDICTION}

For starting the proposed experiment, Firstly Python3+ should be installed with the unstructured dataset. The unstructured dataset for this experiment on crime is taken from the details of police incident reports of Madison City [12]. The used sample data is for crimes of battery, burglary, drug, fraud, injury, intoxicated, robbery, theft, and weapons violations as in figure 2. The data of police incident report details for all crime types is unstructured plain text which presented in text file format as in figure 3.

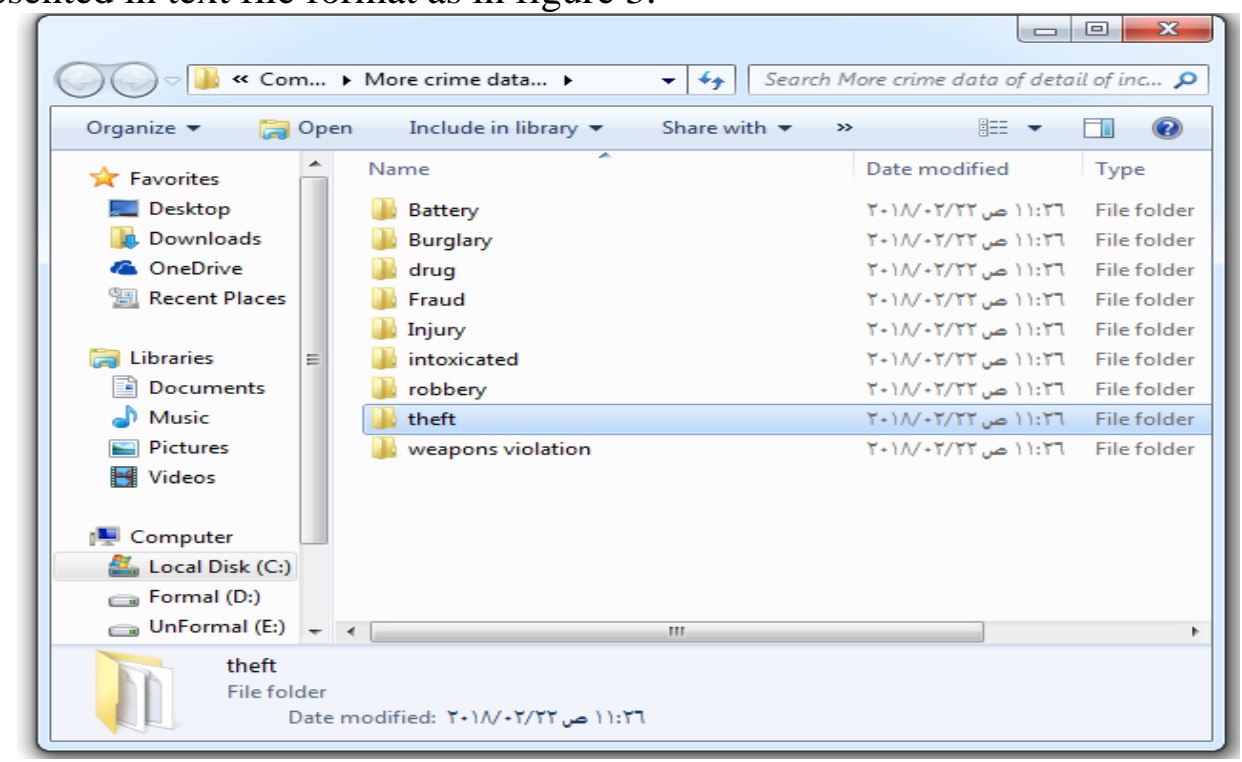

Figure 2 : Types of crime data 


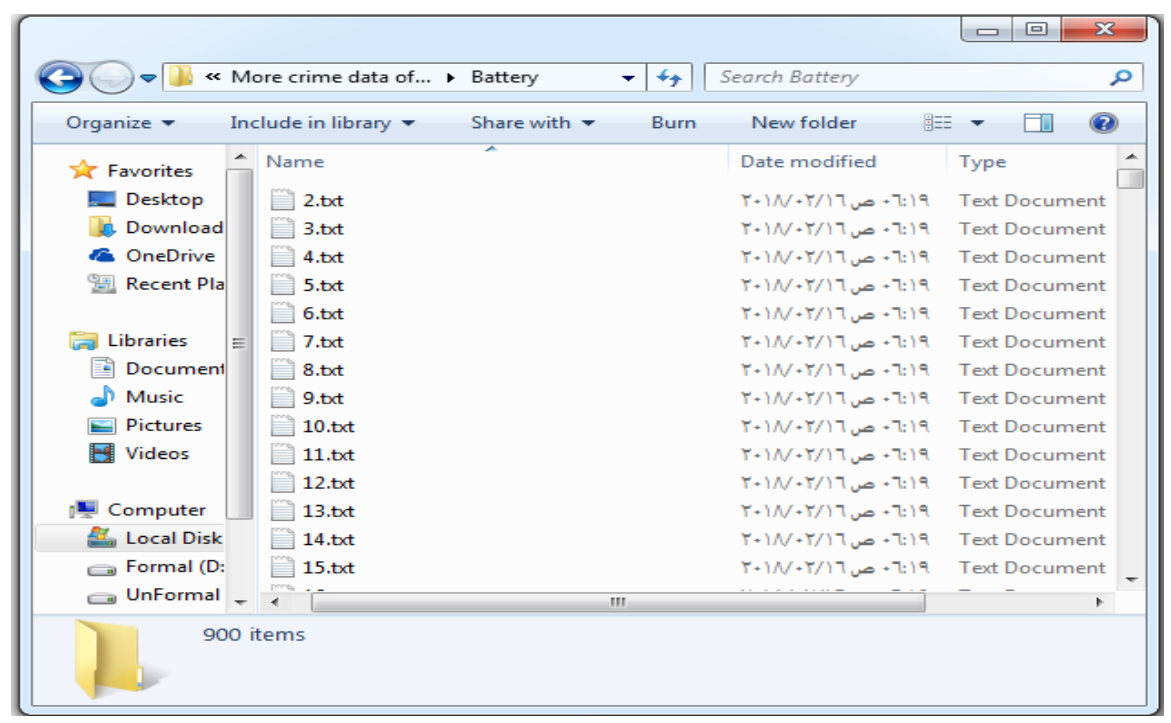

Figure 3: unstructured text format of the data

Building the unstructured text classifier model using Scikit-learn, needs proceeding some of the steps :

\section{○ Step 1: Importing Scikit-learn}

After importing the Scikit-learn, we can begin working the unstructured dataset for the selected text classifier model.

\section{import sklearn}

\section{○ Step 2: Loading the data in Scikit-learn}

The data which are presented in our model as unstructured text, should be loaded in Scikit-learn for operation. Sklearn has a function called load_files that responsible for loading the dataset as text files with categories as subfolder names according the our data that illustrated in figure 2,3 .

\section{from sklearn.datasets import load_files}

○ Step 3: Splitting the input data into training and testing subsets

Sklearn has a function called train_test_split, which divides the input data into training and testing subsets. During this Experiment, the training set is $80 \%$ of total, the rest $20 \%$ is used for the testing purpose.

\section{from sklearn.model_selection import train_test_split}

○ Step 4: vectorizing the training and testing data (Document Presentation)

In order to run the text classification models, the unstructured text documents should be transformed into numerical vectors. The selected model uses uses the Bag-ofwords (BOW) method for representing the text documents using the term frequency weighting schema. This can be done in Sklearn using CountVectorizer function 


\section{○ Step 5: Feature selection}

This step is responsible for reducing the dimensionality of the feature set (the weight matrix was created in step 4), by removing the irrelevant features. The selected text classification model uses Chi square statistic method to be applied in this step. Sklearn supports SelectKBest function that selects features according to highest Chi square scores.

\section{from sklearn.feature_selection import SelectKBest, chi2}

\section{○ Step 6: Constructing Vector Space model}

In this step the vector space model will be constructed according to the selected features in step5 using Term Frequency - Inverse Document Frequency. TF-IDF weighting scheme. TF-IDF can be used in sklearn using TfidfTransformer function.

\section{from sklearn.feature_extraction.text import TfidfTransformer}

\section{○ Step 7: Building the MultiNomial Naive Bayes Classifier}

First, import the MultinomialNB module. Then train the model by fitting it to the train data using fit() function. After we train the model, the trained model can be used to make predictions on any labeled or on unlabeled dataset using the predict() function

\section{from sklearn.naive_bayes import MultinomialNB}

\section{○ Step 8: Evaluating the unstructured text classifier model}

- Calculate the classifier model accuracy:

$$
\text { from sklearn.metrics import accuracy_score }
$$

The accuracy of the model using accuracy_score function is (0.852999340804)

- Classification Report

Return a summary of the precision, recall, F1 score for each crime type as in firgure 4 using the function of classification_report in sklearn.

from sklearn.metrics import classification_report

\begin{tabular}{|c|c|c|c|c|}
\hline & precision & recall & f1-score & support \\
\hline 0 & 0.84 & 0.80 & 0.82 & 186 \\
\hline 1 & 0.95 & 0.80 & 0.86 & 132 \\
\hline 2 & 0.98 & 0.85 & 0.91 & 65 \\
\hline 3 & 0.90 & 0.83 & 0.86 & 69 \\
\hline 4 & 0.93 & 0.67 & 0.78 & 21 \\
\hline 5 & 0.89 & 0.81 & 0.85 & 124 \\
\hline 6 & 0.82 & 0.96 & 0.88 & 512 \\
\hline 7 & 0.79 & 0.78 & 0.78 & 157 \\
\hline 8 & 0.88 & 0.80 & 0.84 & 251 \\
\hline avg / total & 0.86 & 0.85 & 0.85 & 1517 \\
\hline
\end{tabular}

Figure 4: Classification report for the classification model 
- Describing the performance of the classifier model using the confusion matrix:

The performance of a classification model can be summarized by using the confusion matrix as in figure 5, that can be done in sklearn using confusion_matrix function

from sklearn.metrics import confusion_matrix

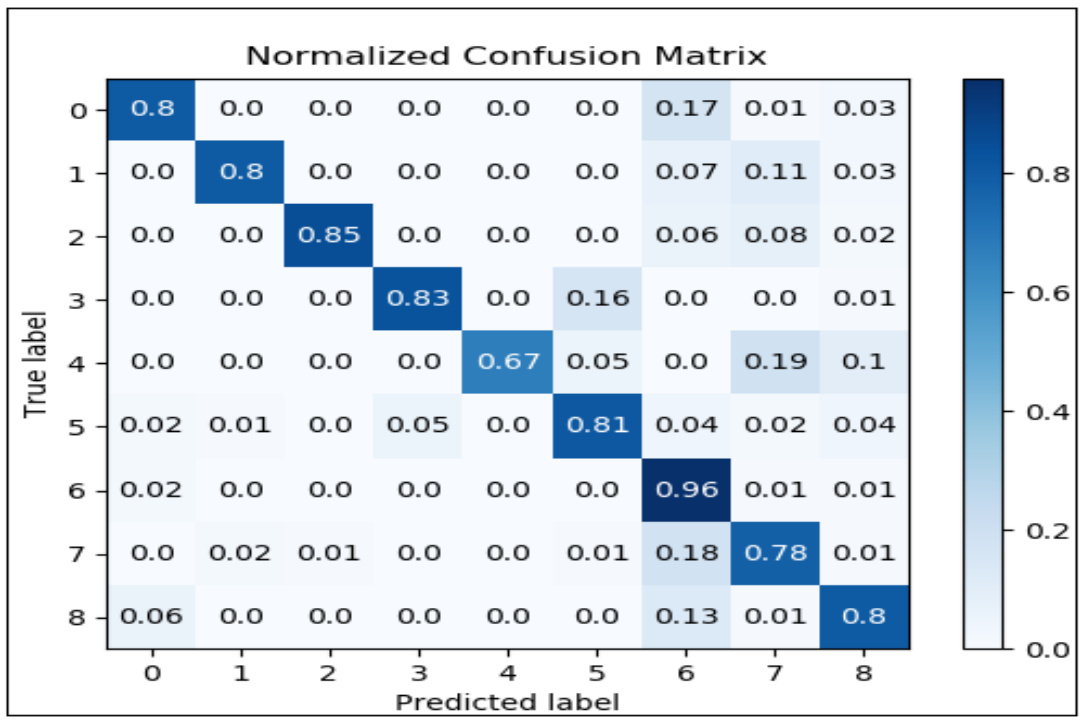

Figure 5: The Confusion Matrix for the classifier model

\section{CONCLUSION}

This paper aims to build an unstructured text classification model using Scikit-learn for applying on the unstructured data of the police incident reports of the city of Madison. The execution results of the model achieved a good result that was very stable and reliable. This prediction can be used to take decision in controlling the crime by comparing the different types to know which crime is less and what crime is more.

\section{REFERENCE}

I. S. Yamuna, N. Sudha Bhuvaneswari, (2012), Data Mining Techniques to Analyze and Predict Crimes, The International Journal of Engineering And Science (IJES), Volume1, Issue 2, Pages 243-247, ISSN: 2319 - 1813 ISBN: 2319 - 1805

II. Waseem Ahmed, TabrezNafis, Siddhartha Sankar Biswas, " Performance Analysis of Naïve Bayes Algorithm on Crime Data using Rapid Miner ", International Journal of Advanced Research in Computer Science, Volume 8, No. 5, May - June 2017

III. Rasoul Kiani, Siamak Mahdavi, Amin Keshavarzi," Analysis and Prediction of Crimes by Clustering and Classification ", (IJARAI) International Journal of Advanced Research in Artificial Intelligence, Vol. 4, No.8, 2015.

IV. Zakaria Suliman Zubi, Ayman Aktaher Mahmmud," Crime Data Analysis Using Data Mining Techniques to Improve Crimes Prevention", Volume 8, 2014

V. Obuandike Georgina N., Audu Isah, John Alhasan," Analytical Study of Some Selected Classification Algorithms in WEKA Using Real Crime Data", International Journal of Advanced Research in Artificial Intelligence, Vol. 4, No.12, 2015 
VI. Tahani Almanie, Rsha Mirza and Elizabeth Lor ," Crime Prediction Based on Crime Types and Using Spatial and Temporal Criminal Hotspots", International Journal of Data Mining \& Knowledge Management Process (IJDKP) Vol.5, No.4, July 2015

VII. Somayeh Shojaee, Aida Mustapha, Fatimah Sidi, Marzanah A. Jabar, "A Study on Classification Learning Algorithms to Predict Crime Status, "International Journal of Digital Content Technology and its Applicatin, Volume7, Number9, May 2013.

VIII. Noora Abdulrahman and Wala Abedalkhader," Knn Classifier and Naive Bayes Classifier for Crime Prediction in San Francisco Context ", International Journal of Database Management Systems (IJDMS) Vol.9, No.4, June 2017.

IX. Cuicui Sun, Chunlong Yao, Lan Shen and Xiaoqiang Yu, "Improving Classification Accuracy Using Missing Data Filling Algorithms for the Criminal Dataset", International Journal of Hybrid Information Technology Vol.9, No.4 (2016), pp. 367-374.

$\mathrm{X}$. Cuicui Sun, Chunlong Yao*, Xu Li, Xiaoqiang Yu, "Detecting crime types using classification algorithms",BioTechnology: An Indian Journal, Volume 10 Issue 24 , 2014, pp. 15451-15457.

XI. Mowafy M, Rezk A, and El-bakry HM," AN Efficient Classification Model For Unstructured Text Document ", American Journal of Computer Science and Information Technology ,2018, Vol.6 No.1:16

XII. https://data-cityofmadison.opendata.arcgis.com/, last accessed on 24/2/2018 